\title{
Conception and development of a dsPIC based polyphase energy metering and power quality analyzer
}

\author{
Anu Mary James ${ }^{1}$ \\ ${ }^{l}$ (Electronics and communication, Mar Athanasius college of engineering, India)
}

\begin{abstract}
The presented paper describes the studies and experimental results conducted about the various aspects of power quality indices measurement as part of the project "the conception and development of a DSPIC based poly phase energy metering and power quality analyzer". The system is developed based on dsPIC30F2023 processor and the ADE7880 Polyphase Multifunction Energy Metering IC with Harmonic Monitoring. Studies are done on the interfacing of the system through UART with the PC using terminal software, interfacing the LCD module, configuring the ADE7880 IC and SPI interface to processor. Development environment is MPLAB IDE, along with the XC16 compiler and the computations are carried out in MATLAB exploiting the UART communication. Analysis and computation of various parameters like the line frequency, rms voltage and current, power factor, voltage sag and power have been done. Use of a dedicated metering has made the system more accurate and incorporation of MATLAB resulted in efficient computation.
\end{abstract}

Keywords - energy metering IC, power factor, power quality analyzer, power quality, SPI, voltage sag.

\section{Introduction}

AC power systems are designed to operate at a sinusoidal voltage of a given frequency and magnitude. Any significant deviation or distortion in the waveform magnitude, frequency or purity is a potential power quality problem[1]. Even though Power quality has been an issue since electrical power was invented, it has only become a well published issue in recent years because of the increased use of equipments and systems that are sensitive to fluctuations.

Most of the industrial motor drives are based on power electronic circuits which form a nonlinear load in a power system characterized by the introduction of a switching action and consequently current interruptions. This behavior provides current with different components that are multiples of the fundamental frequency of the system called harmonics. Current harmonics deteriorate the power factor of the system where as current distortions can produce voltage distortions. When currents with harmonics flow through electrical generation systems and transmission lines, additional distortions take place because of the impedance of the electrical network. For applications in which quality of power supply is important, we have to maintain the quality within particular limits. For that, quality monitoring and correction are needed.

The previous works related to this include the power quality parameters analysis and standardization, in which power quality problems, issues, related international standards, effect of power quality problem in different apparatuses and methods for its correction are, discussed [2]. In the area of developing of a power quality analyzer different approaches have been made which include MATLAB server pages as the core of the system [3], in which a remote area power quality is analyzed using internet and a low bandwidth communication is used but the accuracy and reliability of the parameters will be an issue even though remote monitoring is possible. Another method was using virtual instrumentation and PC [4], in which a PC is utilized to create a virtual monitoring instrument which is a different approach. Another work using a raspberry pi and a GPRS module [5], in which the system is using one of the latest modules and GPRS will definitely increase the range but the use of advanced modules for a power monitoring system is not necessary. Harmonics is one of the most important parameter in power monitoring. Experimental studies are performed in [6], investigating the power consumptions and harmonic absorptions of different types of indoor luminaries.

This work proposes the conception and development of a dsPIC based polyphase energy metering and power quality analyzer for power quality monitoring in which a dedicated energy metering IC is being used and thereby making the system more accurate and reliable compared to other signal processing techniques implemented using processors.

\section{Design and realization of power quality metering system}

The functional block diagram of the power quality analyzer developed to monitor and display the power quality parameters of any four wire utility electrical power supply at regular intervals is shown in Fig.1.The system has been developed based on the ADE7880 energy metering IC [7] along with the DSPIC2023 microcontroller [8] where as the communication between them is implemented with SPI [9]. 
The main building blocks of the system includes a power supply conditioning and scaling circuit board which incorporates current transformers and potential transformers which are directly connected to the 3 phase AC at one end. The other end is connected to the respective inputs of the energy metering IC. The measured parameters can be displayed in the LCD display or a PC monitor which is accomplished with a UART communication interface.

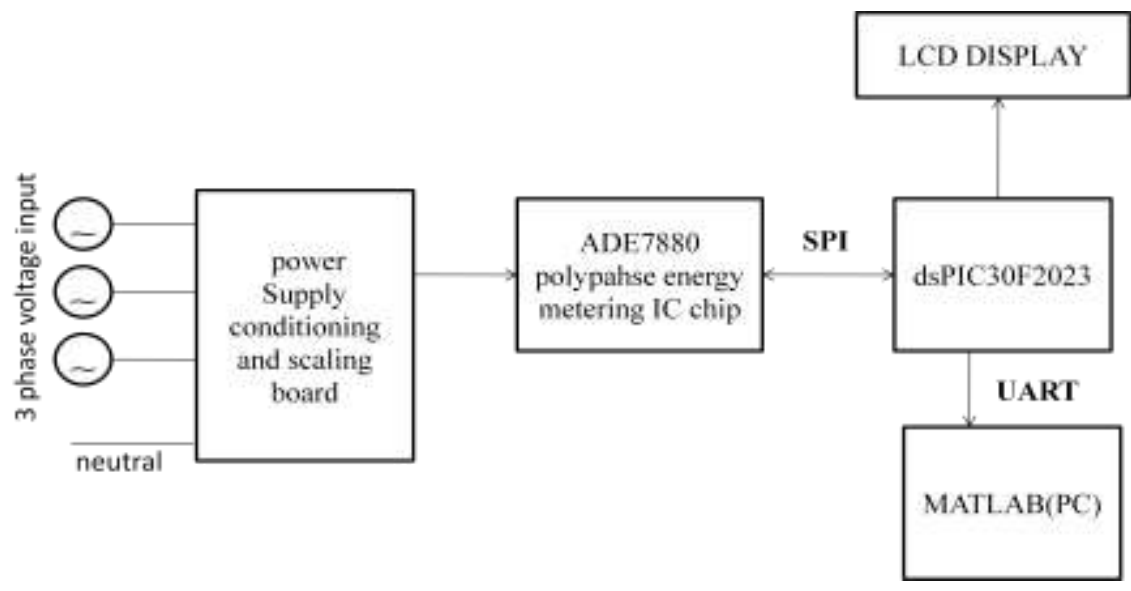

Figure1 functional block diagram of the power quality analyzer based on ADE7880

\section{Serial interfacing of the dsPIC2023 with the MATLAB}

Due to the limitation in the memory of the processor used and for more computation efficiency and also for minimal loading of the processor the major part of the computations are done using MATLAB. So the processor is serially interfaced to the PC so that computations are done using MATLAB [10] and for doing that the following should be done. The register values of the energy metering IC read by the processor are sent to the PC using the serial communication established. The baud rate must be set always and always Check the Terminator, flow control (defaults usually ok), Parity (defaults usually OK), databits (defaults usually OK) and byteorder. For this a simple single line code was written at the beginning of the MATLAB code.

\section{Measurement of three phase supply parameters}

The system is designed to measure various power quality related parameters as mentioned below. The system is designed to give a maximum voltage of $500 \mathrm{~V}$ and the current calibration has been done by connecting a $100 \mathrm{~W}$ bulb as load. Fig. 2 shows the basic circuit diagram used for testing.

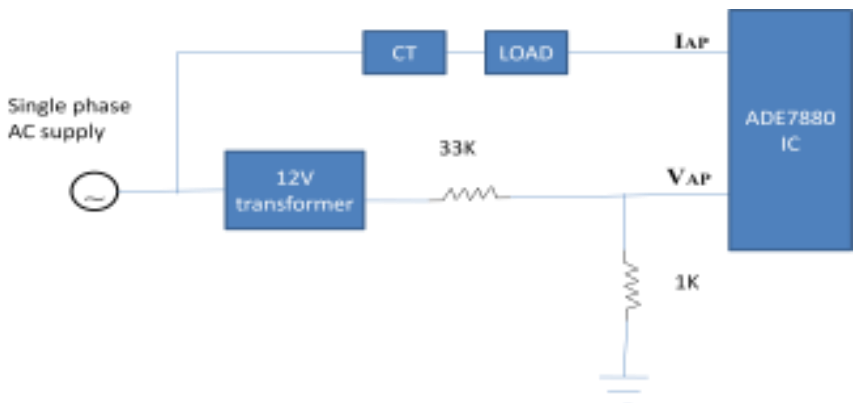

Figure 2 testing circuit for the computation of power quality parameters

4.1 Period measurement

The ADE7880 provides the period measurement of the signal in the voltage channel. The period of each phase voltage is measured and stored in three different registers APERIOD, BPERIOD, and CPERIOD [7]. Equation (1 )is used to compute the line period and frequency using the period registers:

$$
f_{L}=\frac{256 \mathrm{E} 3}{\text { PERIOD }[15 ; 0]}[\mathrm{Hz}]
$$




\subsection{Voltage channel rms calculation}

The voltage channel rms value is processed from the samples obtained from the voltage channel. The voltage rms values are stored as signed 24-bit values into the registers AVRMS, BVRMS, and CVRMS . With the specified full-scale analog input signal of $0.5 \mathrm{~V}$, the ADC produces an output code that is approximately $\pm 5,326,737$. The equivalent rms value of a full-scale sinusoidal signal is $3,766,572$. Fig. 3 shows the various steps involved in computing the rms voltage along with the required communications.

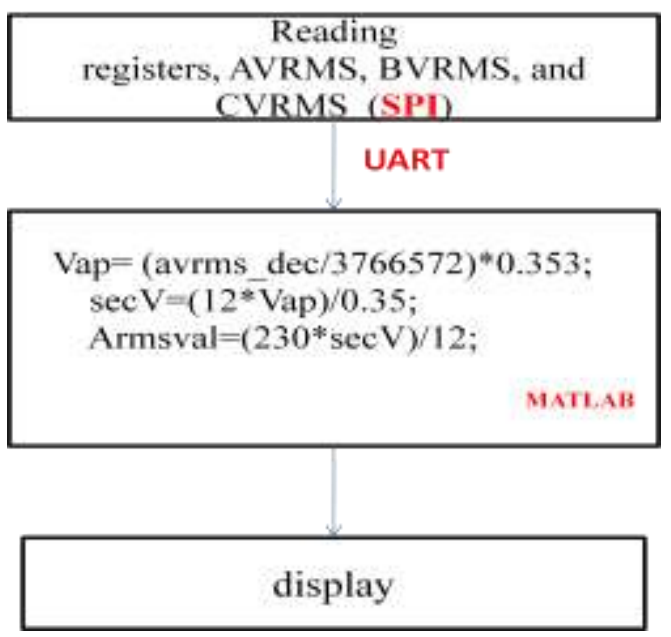

Figure 3 flow diagram of rms voltage computation

\subsection{Current rms calculation}

The current channel rms value is processed from the samples obtained from the current channel. The current rms values are stored as signed 24-bit values into the AIRMS, BIRMS, CIRMS registers. Fig. 4 shows how the RMS value of the phase current is computed and calibrated in this system with a purely resistive load connected (a 100W bulb).

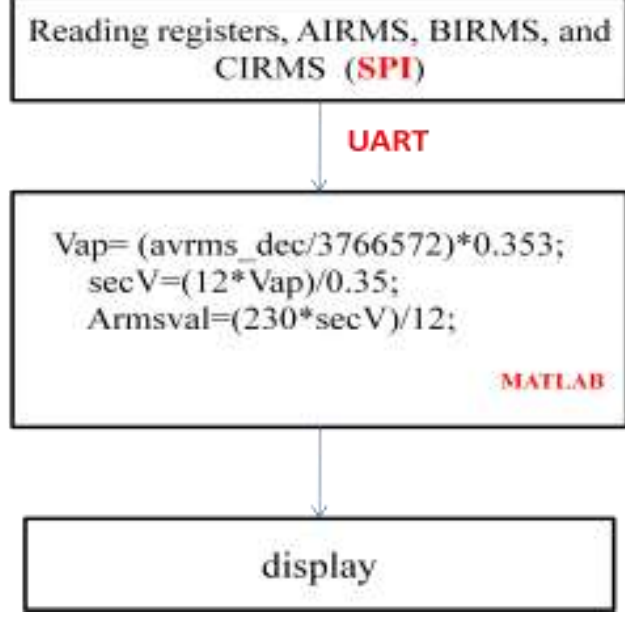

Figure 4 flow diagram of rms current computation

\subsection{Peak detection and computation}

The ADE7880 records the maximum absolute values reached by the voltage and current channels over a certain number of half- line cycles and stores them into the less significant 24 bits of the VPEAK and IPEAK 32-bit registers. Composition of IPEAK[31:0] and VPEAK[31:0] Registers are as shown in fig.5 and fig. 6 shows the flow diagram of peak detection and computation. 


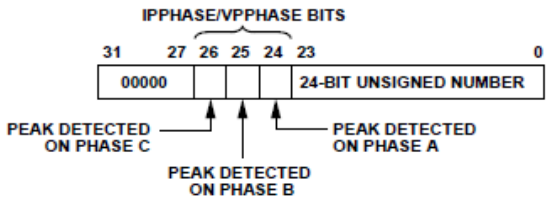

Figure 5 composition of IPEAK[31:0] and VPEAK[31:0] Registers

\section{Writing registers PEAKCYC,MMODE}

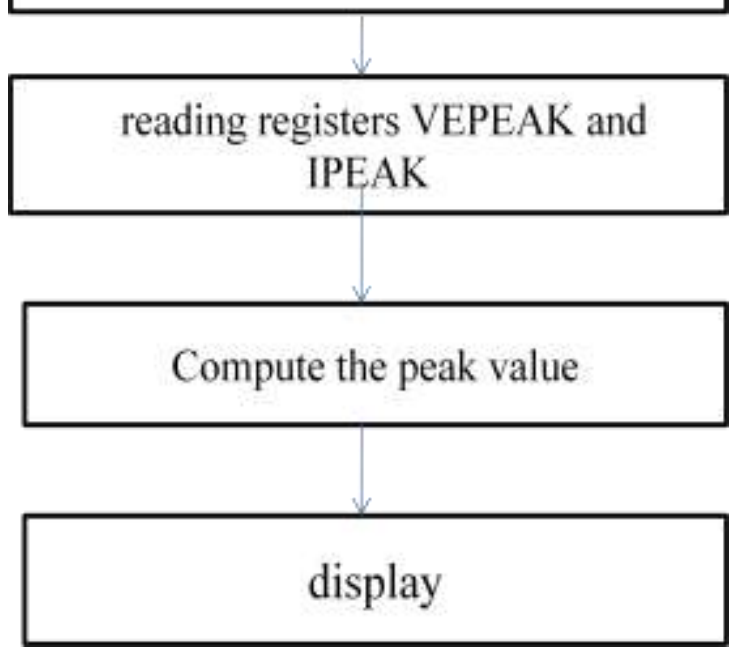

Figure 6 flow diagram of peak detection and computation

\subsection{Phase voltage sag detection}

Voltage sag happens when the rms voltage decreases between 10 and 90 percent of nominal voltage for one-half cycle to one minute. The following steps are to be done to detect a voltage sag in any of the 3 phases A, B, or C. A threshold voltage is set in the sag level register (SAGLVL) for number of half-line cycles set in SAGCYC register which represents the number of half-line cycles the phase voltage must remain below or above the level indicated in the SAGLVL register. Bit 16 (SAG) in the STATUS1 register is set to 1 when sag condition occurs in any of the 3 phases and to know in which phase we have a sag, read the Bits VSPHASE in the PHSTATUS register. Fig. 7 shows the flow diagram for SAG detection.

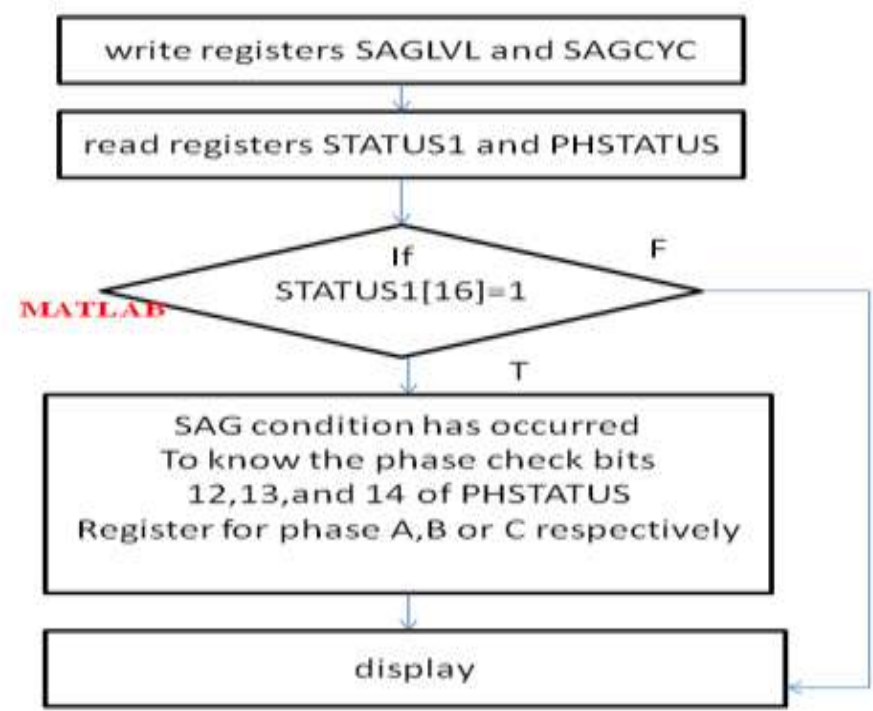

Figure 7 flow diagram of sag detection 


\subsection{Overvoltage and overcurrent detection}

When the voltage in a circuit or part of it is raised above its upper design limit, this is known as overvoltage can be transient or permanent leading to surge. Here the system was designed for a maximum peak of $500 \mathrm{~V}$. When the input is greater than $500 \mathrm{~V}$ the system will detect an overvoltage. Similar analysis has done for overcurrent, and Fig.8 shows the steps involved in overcurrent and overvoltage detection using ADE7880.

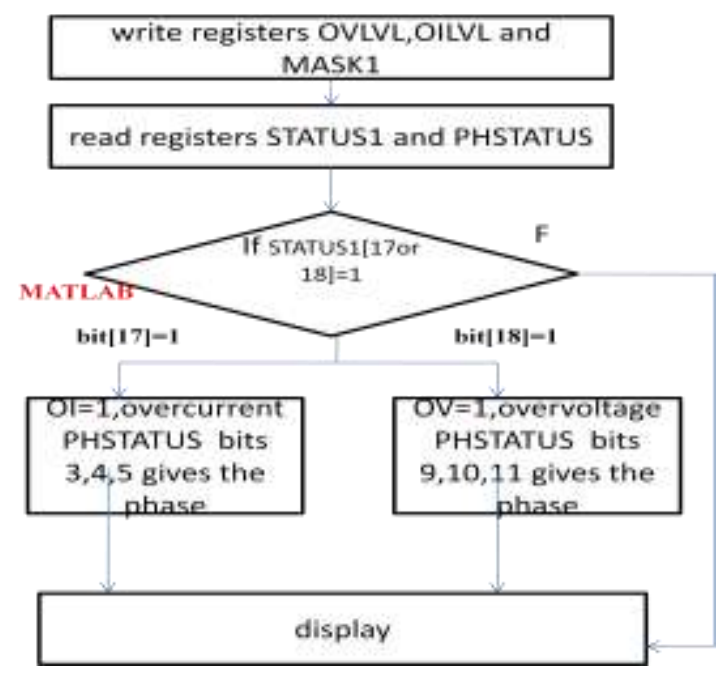

Figure 8 flow diagram of overvoltage and overcurrent detection

\subsection{Power factor calculation}

There are three methods for measuring power factor using ADE7880 of which two are considered for the analysis.

\subsubsection{Power factor computation using the displacement angle}

The ANGLE0 register would give the displacement value which is shown in Fig.9 and the following computation as shown in the flow diagram is used to obtain the power factor. The computation of power factor is done as shown in the following diagram explained in Fig. 10.

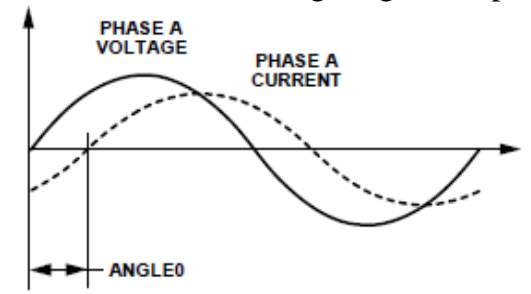

Figure 9 delay between Phase A Voltage and Phase A Current is Stored in the ANGLE0 Register

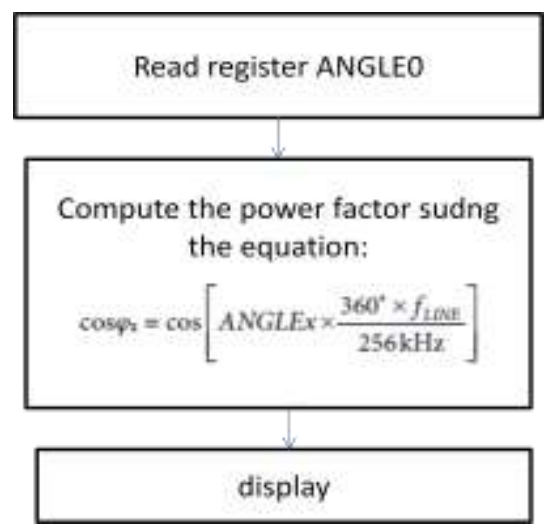

Figure 10 flow diagram of power factor computation using ANGLE0 register 


\subsubsection{Reading directly from the registers APF, BPF and CPF}

The power factor is defined as the ratio of the total active power flowing to the load to the apparent power (2). The ADE7880 provides a power factor measurement on all phases simultaneously. These readings are provided into three 16-bit signed registers, APF, BPF, CPF for Phase A, B and C respectively. Reading these registers and a corresponding evaluation will give the power factor.

$P . F=($ sign of undamental reactive power $) \times \frac{\text { abs (total active power })}{\text { Apparent power }}$

\subsection{Active power calculation}

The ADE7880 computes the total active power on every phase. Total active power considers in its calculation fundamental and all harmonic components of the voltages and currents. In addition, the ADE7880 computes the fundamental active power, the power determined only by the fundamental components of the voltages and currents. The equation of active power is as given in (3).Active power is computed as:

$$
\operatorname{Vrms} \operatorname{Irms} \operatorname{Cos}(\varphi) \text {. }
$$

\section{Hardware realization}

The hardware design of the power quality analyzer system includes 3 main blocks namely the power supply conditioning and scaling board, the ADE7880 IC board and the DSPIC30F2023 development board. The entire setup of the system for testing and verification is shown in Fig.11.

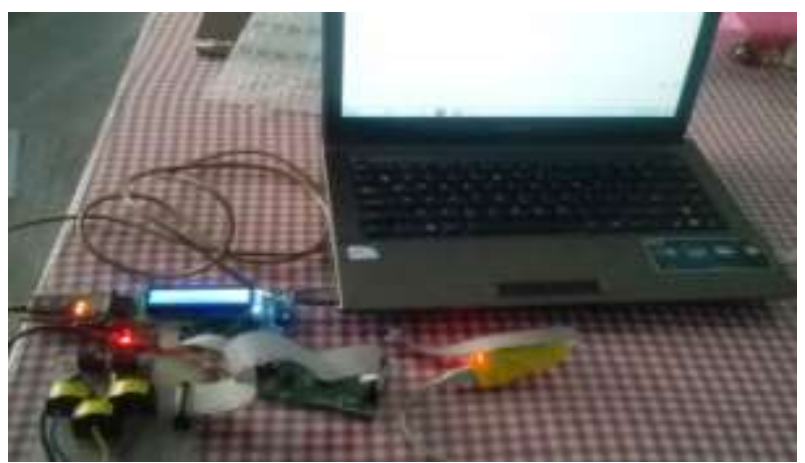

Figure 11 testing setup of the developed system

A power supply conditioning and scaling circuit is at the supply end where the required conditioning and scaling (stepping down of voltage) etc takes place. It consists of 3 step down transformers and current transformers. A snapshot of the board is shown in Fig. 12.

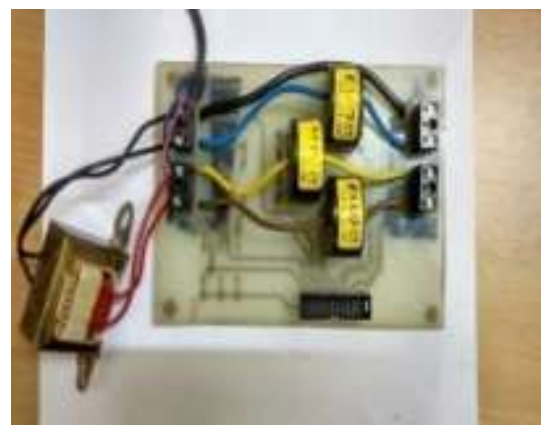

Figure 12 the power supply conditioning and scaling board

The development board for the ADE7880 working at a supply voltage of 3V is shown in Fig.13. 


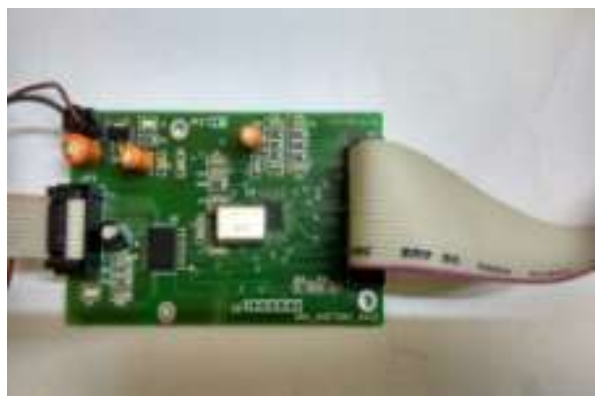

Figure 13 ADE7880 IC Board with Isolation

\section{CONCLUSION}

The monitoring and analysis of certain power quality parameters are described in this paper. The hardware realization of the power quality meter built around ADE7880 and dsPIC as well as the algorithm for the computation of the various parameters monitored are described. SPI interfaces between energy metering IC and processor, as well as UART interface to PC have been used to pursue the computation in MATLAB. The system developed can monitor and display the power quality parameters of any four wire utility electrical power supply at regular intervals. The advantages of the system include an accurate measurement system for power quality parameters. Since the results are computed using MATLAB the computational efficiency is very high and additional matlab features can also be exploited for a detailed analysis. The drawback of the system is that always a proper serial communication must be maintained between matlab and the processor because if this fails then the MATLAB software must be restarted. The future scope of this system is to extend this to an Internet of Things (IoT) based system by including a communication module like the GPRS and monitoring power from a distance.

\section{REFERENCES}

[1] Roger. C. Dugan, Mark. F. Mc Granagham, Surya Santos, H. Wayne Beaty, 'Electrical Power Systems Quality' McGraw Hill.

[2] S.Khalid and B. Dwivedi, Power quality issues, problems, standards \& their effects in industry with corrective Means, International Journal of Advances in Engineering \& Technology, 1(2),2011,1-11.

[3] B. Vural, A. Kizil and M. Uzuno־Glu, A power quality monitoring system based on MATLAB server pages, Turk J Elec Eng \& Comp Sci, 18(2), 2010

[4] Z. Kokolanski, M. Srbinovska, A. Simevski, C. Gavrovski and V. Dimcev, Power Quality Monitoring and Power Measurements by Using Virtual Instrumentation, ELECTRONICS, VOL. 13(1), 2009, 70-76.

[5] P. Yadav and V. N. Chavda, Development of power monitoring and analysis system based on web server, International Journal of Engineering Development and Research,2(2), 2014,1840-1843

[6] A.Dolara and S. Leva, Power Quality and Harmonic Analysis of End User Devices, Energies, 5, 2012, 5453-5466

[7] ADE77880-Poly Phase Multifunction Energy Metering IC with Harmonic Monitoring Analog Devices, Data sheet, www.analog.com

[8] dsPIC30F1010/202X Enhanced Flash16-bit Digital Signal Controller Microchip Technology Inc., 2014.

[9] dsPIC30F Family Reference Manual-High-Performance Digital Signal Controllers Microchip Technology Inc., 2006.

[10] Matlab Serial Communication Tutorial Esposito, http://www.usna.edu/Users/weapsys/esposito/, 2009, 1- 16 